\title{
Research Paper: A Survey on Cognitive Functions of Students With Stuttering
}

\author{
Morteza Farazi ${ }^{1 \oplus}$, *Kambiz Kamkary², Akram Hassanzade Noghani ${ }^{3}$
}

1. Department of Speech Therapy, University of Social Welfare and Rehabilitation Sciences, Tehran, Iran. 2. Department of Education and Consultation, Islamshahr Branch, Islamic Azad University, Tehran, Iran.

3. Department of Education and Consultation, Faculty of Literature Humanities and Social Sciences, Science and Research Branch, Islamic Azad University, Tehran, Iran.

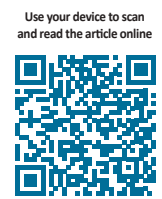

Cittat on: Farazi M, Kamkary K, Hassanzade Noghani A. [A Survey on Cognitive Functions of Students With Stuttering (Persian)]. Archives of Rehabilitation. 2018; 19(2):160-167. http://dx.doi.org/10.32598/rj.19.2.160

doi): http://dx.doi.org/10.32598/rj.19.2.160

Received: 01 Nov 2017 Accepted: 28 Mar 2018

Keywords:

Stuttering, Stu-

dents, Intelligence test, Stanford-Binet Intelligence Scale

\section{ABSTRACT}

Objective Stuttering is one of the most common speech disorders that generate many complications in children and adults. This disorder involves behavioral, cognitive and emotional interactions. So, the purpose of the current study is to investigate the cognitive functions of students with stuttering.

Materials \& Methods A descriptive study, comprising of 30 students (8 females and 22 males) from different educational levels (preschool, elementary and junior high school), was conducted. Study subjects were selected by an available sampling method from the students referred to speech therapy clinics and psychological counseling and rehabilitation centers in Tehran city. The subjects were assessed by using Tehran-Stanford-Binet intelligence scale. This tool consists of verbal and non-verbal domains; each of the fields has five subscales of fluid reasoning, knowledge, quantitative reasoning, visual-spatial processing and working memory. It also has the ability to deliver $8 \mathrm{IQ}$ s, including IQs of fluid reasoning, knowledge IQ, quantitative reasoning IQ, visual-spatial processing IQ, working memory IQ, verbal IQ, nonverbal IQ, and general IQ in the age range of 2 to 85 years. Credit coefficients exceed $90 \%$ among the ten subscales of this intelligence test. The duration of the test for each person varied from quarter of an hour to one and a half hours. Average scores of the experiment were acquired, and data analysis was performed with SPSS software version 19. Student's t-test was conducted to compare, review and analyze the theoretical averages obtained from standardization processes of the test.

Results The results of the study indicated that the general IQ and verbal IQ of the students with stuttering was above the average (expected level) $(P<0.001)$ and their nonverbal IQ was average $(P<0.202)$. The fluid reasoning IQ ( $P<0.001)$, quantitative reasoning IQ $(P<0.020)$, knowledge IQ $(P<0.037)$, and visual-spatial processing IQ $(P<0.001)$ of the students were above average while the working memory IQ was average. Notably, there was no significant difference at the $\alpha=0.01$ level between theoretical meanings and the experimental mean of working memory IQ and non-verbal IQ in stuttering students; these two IQs in these students were moderate in society. Children with stuttering showed a weaker performance in some aspects of working memory compared to normal children of their age. In describing the working memory of children with stuttering, we can mention the role of phonological input and output reservoirs. Children with stuttering are more likely to have phonological input reservoirs, which is very important in the speech and working memory process. Therefore, the ability of this reservoir leads to strong repetition of words, and it seems that although these children have difficulty expressing words and speech, their memory function is increased from moderate to expected level because of the frequency of words in their phonological input reservoir.

Conclusion Findings from this study showed that working memory IQ of students with stuttering is weaker than the IQs of intelligence (four constituent agents); in spite of speech disorder, general IQ and IQs of fluid reasoning, quantitative reasoning, knowledge and visual-spatial processing of these students with stuttering are above average, and they have a good background for teaching and learning. 


\title{
بر رسى كنش هاى شناختى دانش آموزان داراى لكنت
}

\author{
مرتضى فرازى 'ه. •كامبيز كامكارى"، اكرم حسنزاده نوغانى

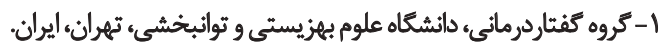

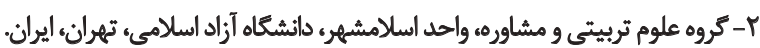

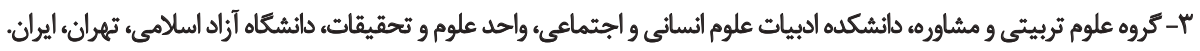

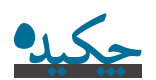

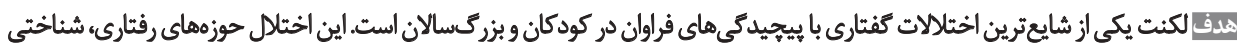

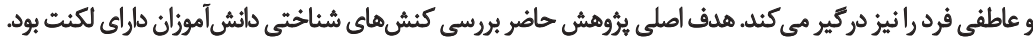

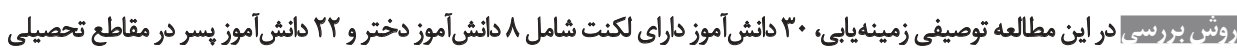

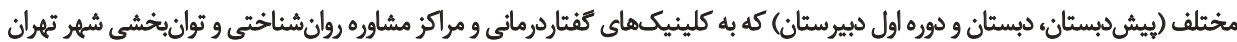

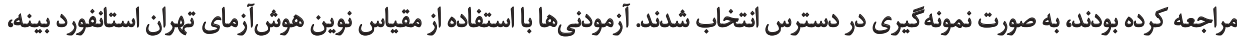

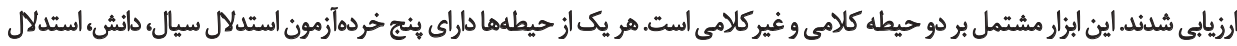

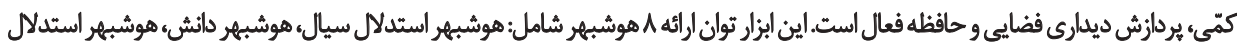

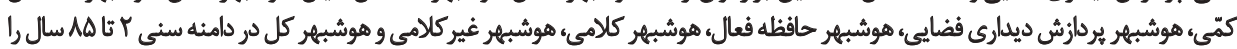

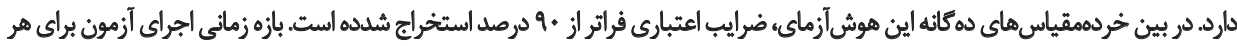

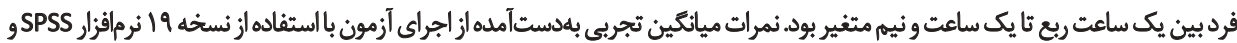

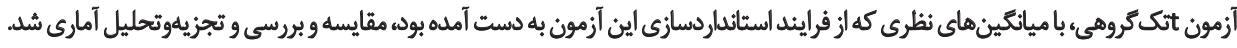

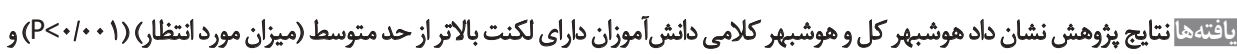

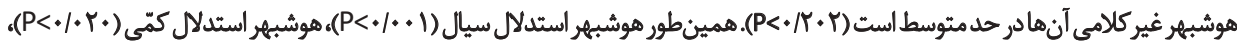

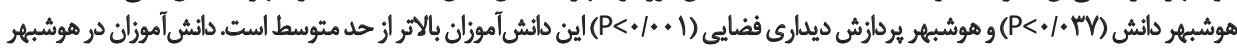

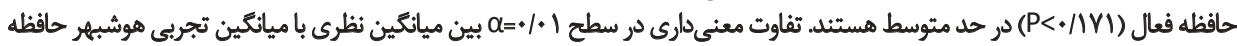

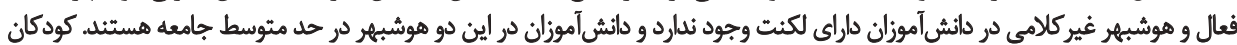

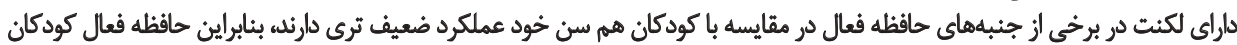

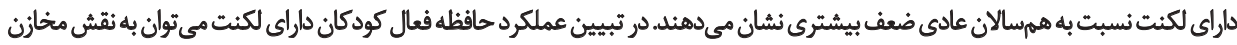

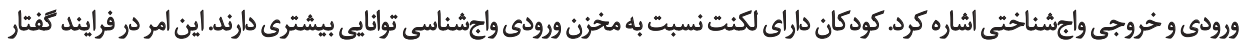

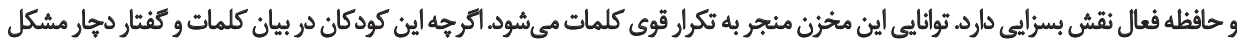

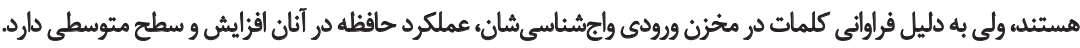

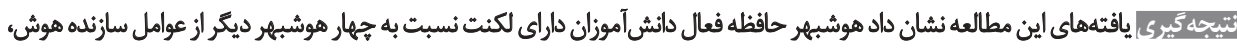

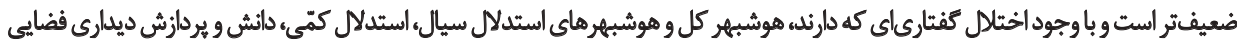

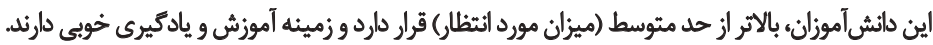

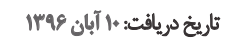
تاريخ بذيرش: A+ قروردين

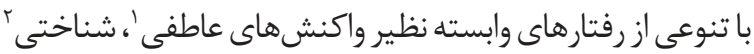

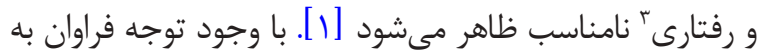

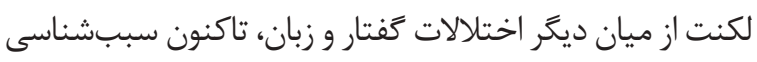

\section{Affective response}

2. Cognitive response

3. Behavioral response مقإd

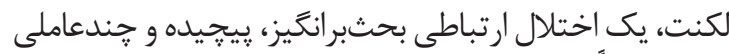

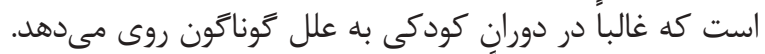

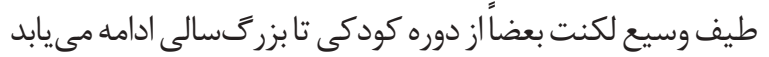
و به عنوان يك اختلال مزمن و يايدار روانى گفتار مطرح است كن كه

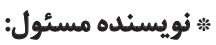
دكثر كامبيز كامكارى

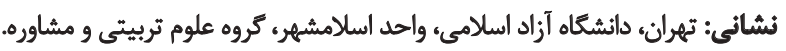

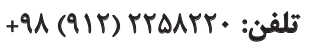
راياثامه: shohreh_skokrzadeh@yahoo.com 


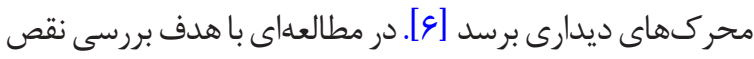

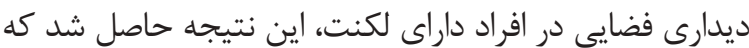

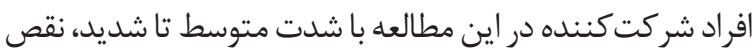

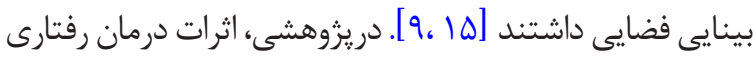

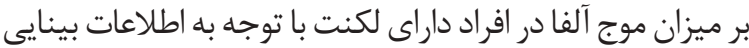

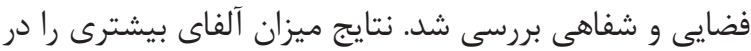

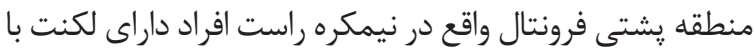

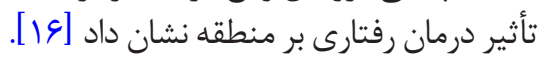
يكى ديخر از كنشهاى شناختى، الدانش" است كه عوامل

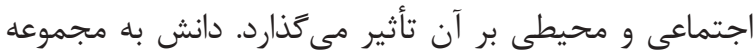

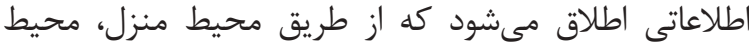

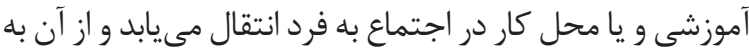

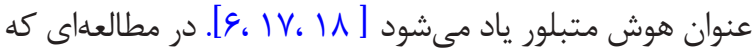

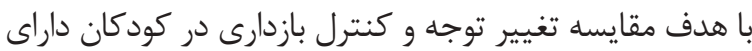

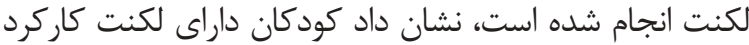

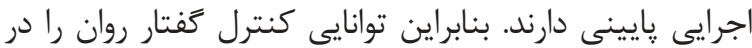

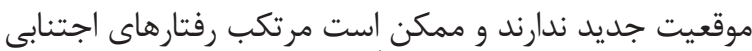
شوند. رفتارهاى اجتنابى معمولا عادت است و و باز تابتى از از افزايش

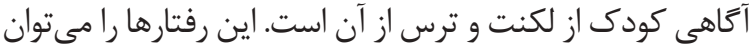

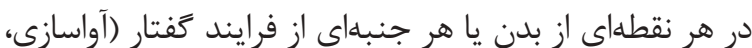

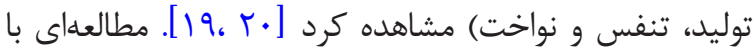

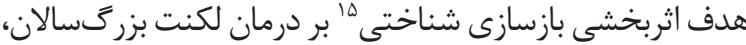

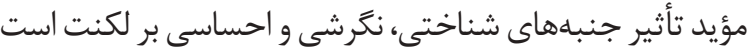
[rI, Yr]

استدلال سيال نيز يكى ديخًر از كنشهاى مورد توجه است كه

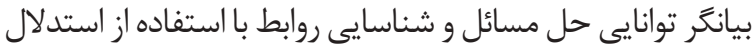

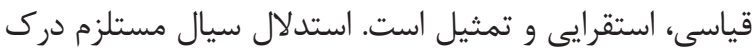

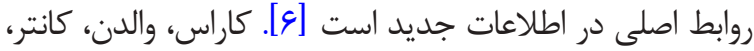

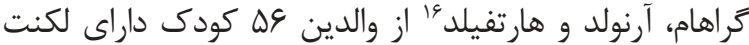

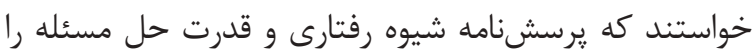

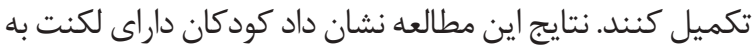

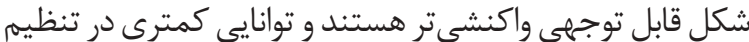

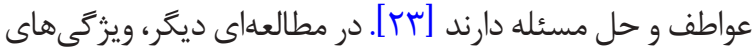

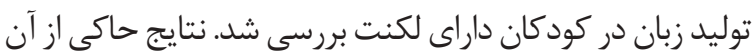

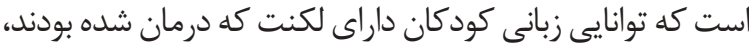

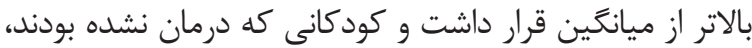

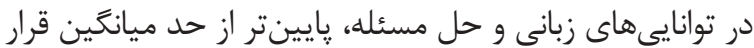

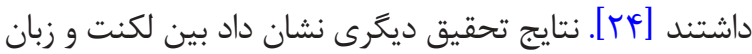

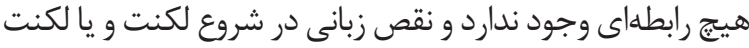

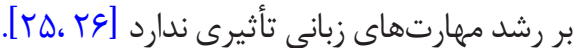

استدلال كمّى نيز يكى ديخر از كنشهاى شناختى قابل توجه

\section{Cognitive restructuring}

16. Karrass, Walden, Conture, Graham, Arnold, \& Hartfield

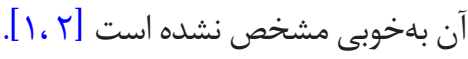

در اين زمينه، مدل كالمز پينج عامل مرتبط به هم ازجمله

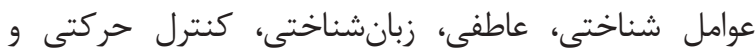

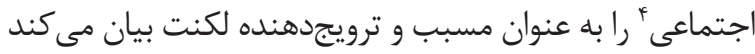

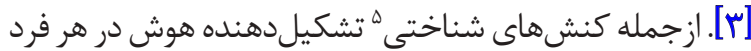

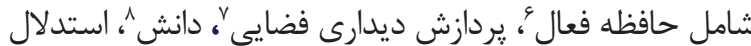

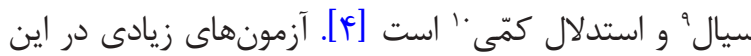

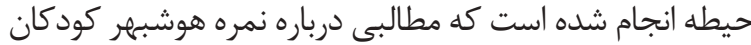

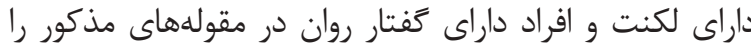

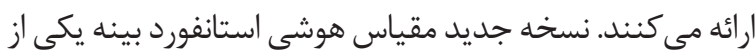

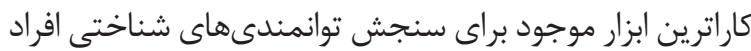

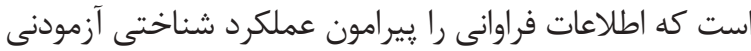

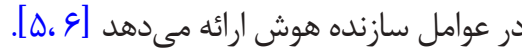

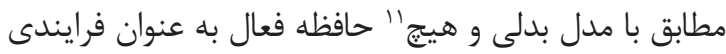

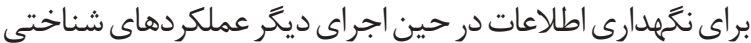

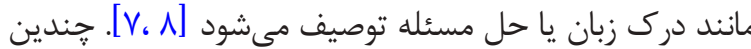

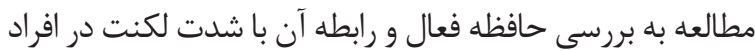

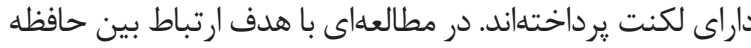

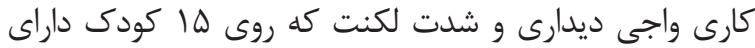

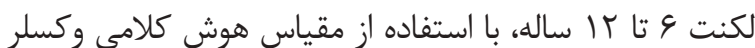

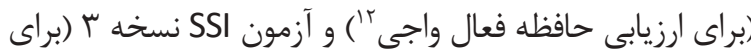

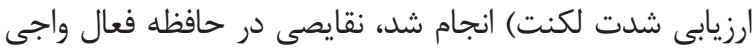

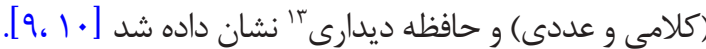

در مطالعه ديخرى كه به بررسى توانايىهاى حافظه فعال كودكان

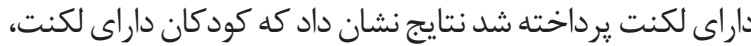

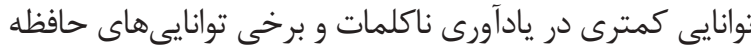

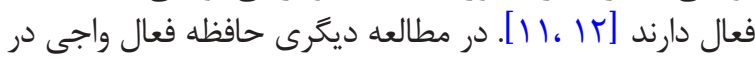

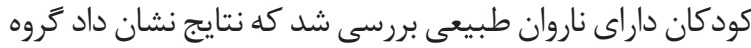

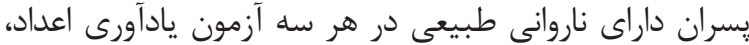

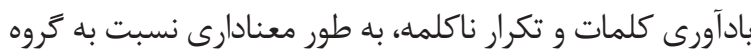

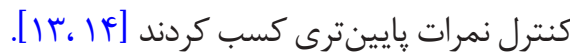

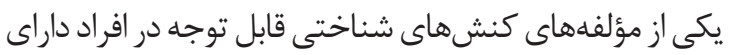

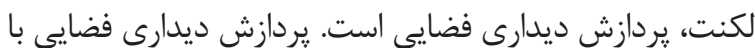

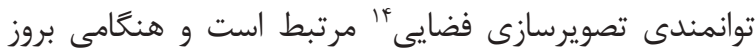

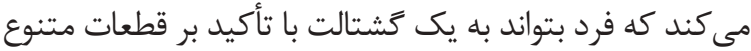

4. Cognitive, Affective, Linguistic, Motor, \& Social (CALMS)

5. Cognitive actions

6. Working memory

7. Visual -spatial processing

8. Knowledge

9. Fluid reasoning

10. Quantitative reasoning

11. Baddeley and Hitch

12. Phonological working memory

13. Visual memory

14. Spatial imaging 


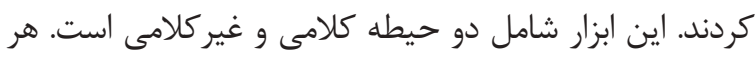

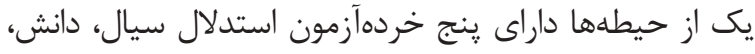

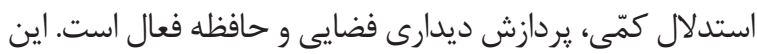

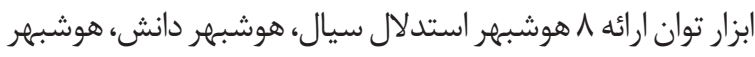

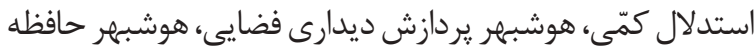

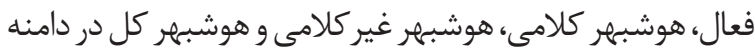

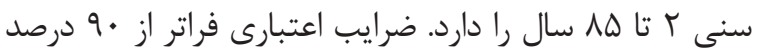

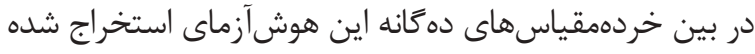

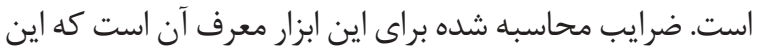

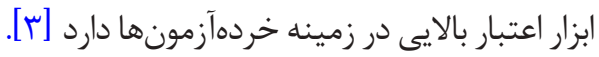

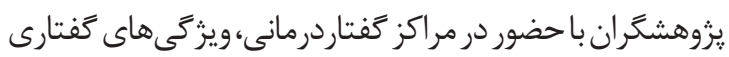

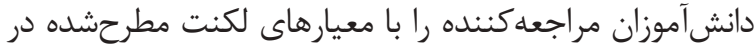

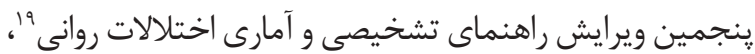

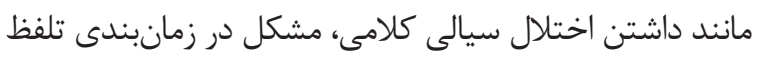

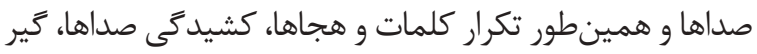

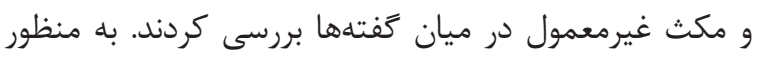

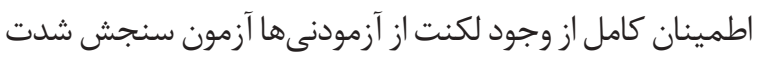

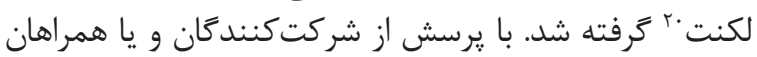
آنها مشخصات فردى تكميل شد.

يس از معاينات اوليه به لحاظ ورود اين افراد به مطالعها،

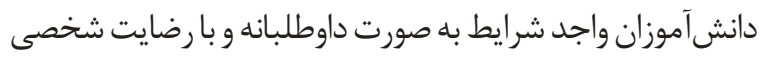

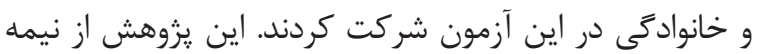

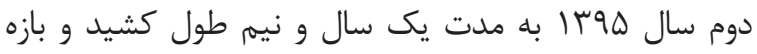

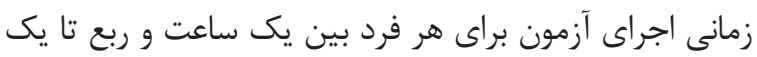

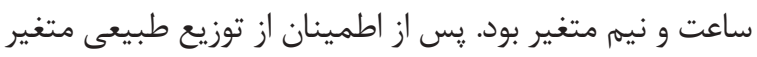

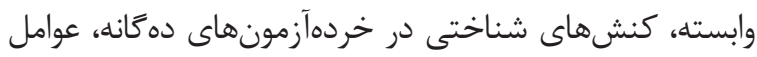

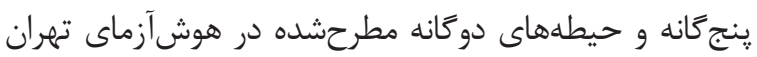
استانفورد بينه، با استفاده از نرمافز إزار

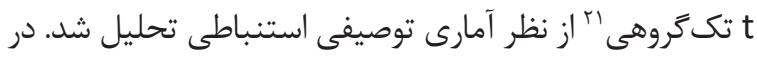

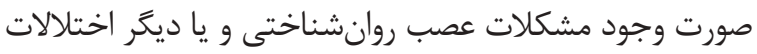

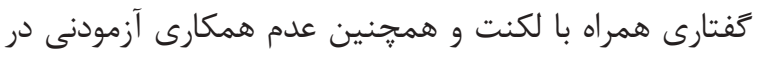
طول تحقيق، فرد از مطالعه خارج مىشند.

يافتهها

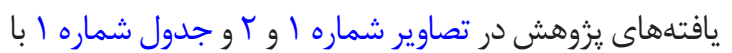

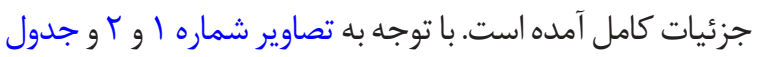

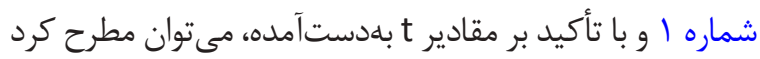

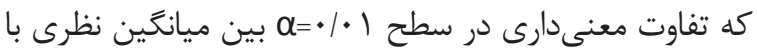

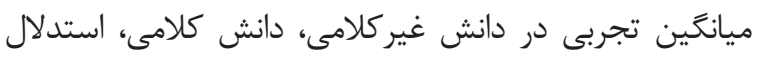

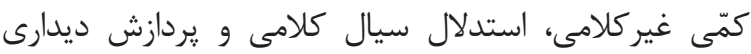

19. Diagnostic and Statistical Manual of Mental Disorders, $5^{\text {th }}$ Edition (DSM-5)

20. Stuttering Severity Instrument, $4^{\text {th }}$ Edition (SSI-4)

21. One sample t-test
در افراد داراى لكنت است كه با فرايند استدلال نيز مرتبط است.

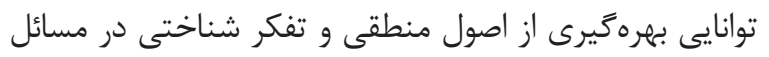

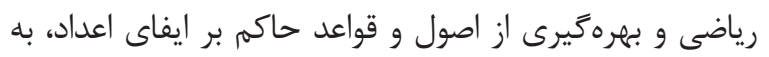

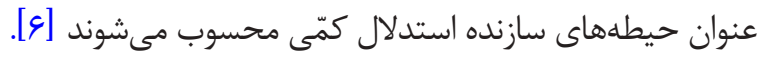

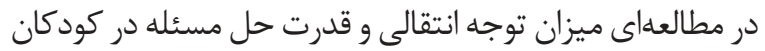

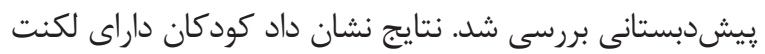

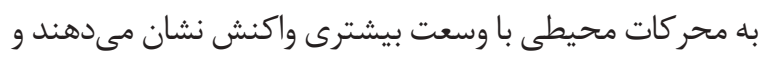

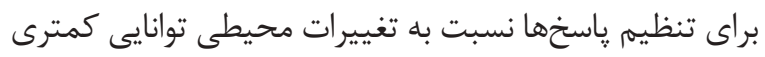
دارند[rV]

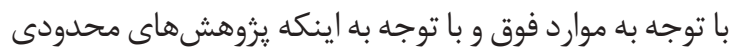

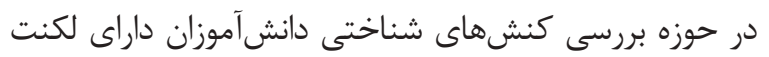

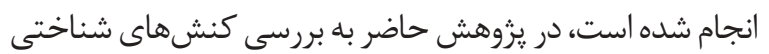

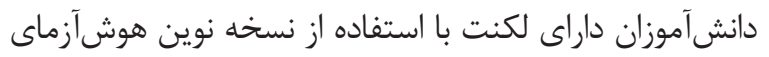

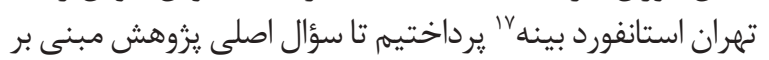

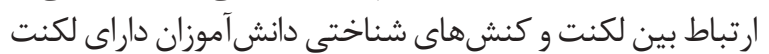

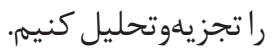

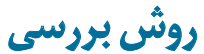

جمعيت مطالعهشده در اين تحقيق دانش آموزان تمامى مقاطع

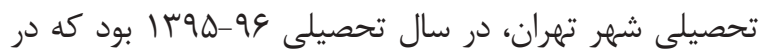

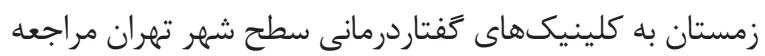

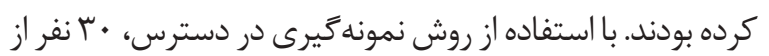

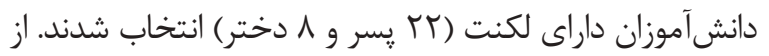

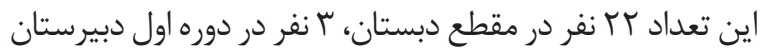

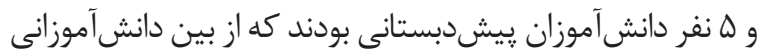

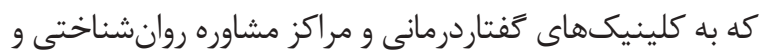

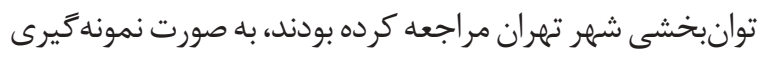

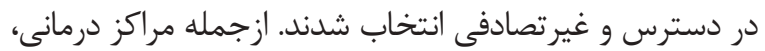

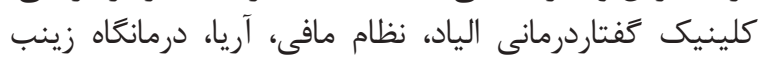

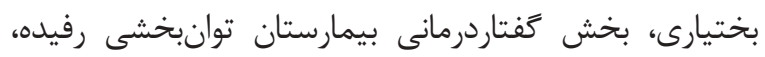

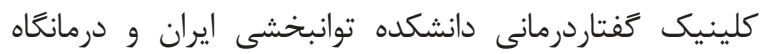
كيمياكر بود.

براى تعيين حجم نمونه از مطالعات مشابه استفاده شد.

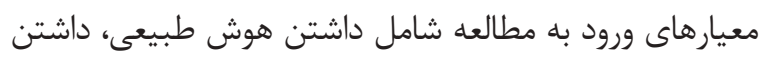

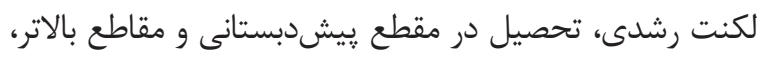

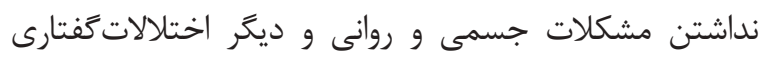

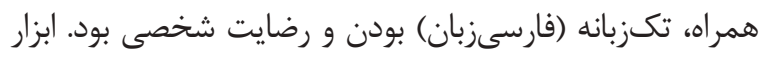

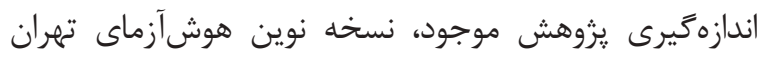

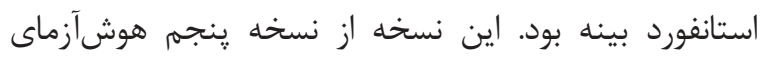

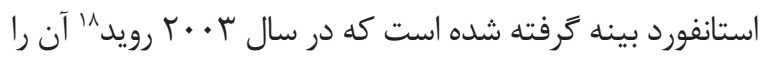

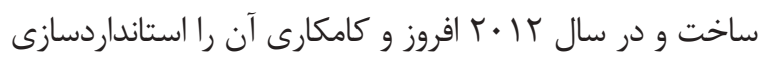

17. Tehran-Stanford Binet- intelligence scale 18. Roid 
جدول ا. t تك كروهى براى بررسى كنش هاي شناختى در دانش آموزان داراي لكثت

\begin{tabular}{|c|c|c|c|c|c|c|}
\hline 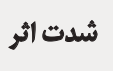 & سطح معنى ذارى & آزادجي & 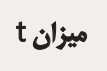 & ميانكين تجربى & مياتكين نظرى & هوشبهر \\
\hline.$/ 18$ &.$/ N T \Delta$ & $\Delta q$ & $V / \Delta \Delta$ & $1 . / N$ & 1. & استدلال سيال غيركلامى \\
\hline 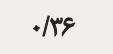 & $\% 1 . .1$ & $\Delta 9$ & $A n^{\circ}$ & $\mid r / E g$ & 1. & استدلال سيال كلامى \\
\hline.$/ M A$ & $.1 . .1$ & $\Delta q$ & $-6 / 99$ & $N r^{m}$ & 1. & دانش غير كلامى \\
\hline$\cdot / \pi$ & .1 .01 & $\Delta q$ & $V / \cdot 1$ & $\mid r / T r$ & 1. & دانش كلامى \\
\hline זית & $\%$ & $\Delta 9$ & $r / r$. & 111.8 & 1. & استدلال كتمى غير كلامى \\
\hline $.1+1$ & .MFE & $\Delta 9$ &.$/ 4 \Delta$ & 1.11 & 1. & استدلال كتى كلامى \\
\hline$+/ F$ & . & $\Delta q$ & V/r & $1 . / \%$ & 1. & يردازش ديلارى فضايع غيركلامى \\
\hline$+N$ & $+1 *+1$ & $\Delta q$ & $8 / \pi$ & $11 / \%$ & 1. & يردازش ديدارى فضايع كلامى \\
\hline$+/ 19$ &.$/ 1 m e$ & $\Delta q$ & Var & $10 / 0$ & 1. & حافظه فعال غير كلامى \\
\hline.$/$ &.$/ 0 M r$ & $\Delta q$ & . & $1 . / 4$ & 1. & هافظط فعال كلامى \\
\hline.$r q$ & .10 .1 & $\Delta q$ & $\Delta / T \Delta$ & $11 . / 14$ & 1.0 & هوشبهر الستدلال سيال \\
\hline.$/ 19$ &.$/ . r r$ & $\Delta q$ & 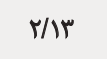 & 1. r/Fe & 1.0 & هوشيهر دانش \\
\hline$\cdot r$ &.$/ \%$. & $\Delta q$ & $r / \% q$ & $1 \cdot r / \cdot r$ & 1.0 & هوشيهر استدلال كمّى \\
\hline.$/ \pi$ & .1 .01 & $\Delta q$ & r/Te & $1+p / e 8$ & 1.0 & هوشبير يردازش ديدارى فضايى \\
\hline.$/ 10$ &.$/|n|$ & $\Delta 9$ & $1 / \pi 1$ & $1 . r / 14$ & 1.0 & هوشيهر حافظه فعال \\
\hline.$/ \pi$ & $\%$ & $\Delta q$ & g/gV & 1.NTE & 1.0 & هوشبهر كلامى \\
\hline$+/ 10$ & $+R+r$ & $\Delta q$ & $1 / T A$ & $1+1 / 1 /$ & 1.0 & هوتشبهر غير كلامي \\
\hline$+/ T \Delta$ & $+1 .+1$ & $\Delta q$ & $\% \% \Delta$ & I. & $1 .$. & هوشبهر كل \\
\hline
\end{tabular}

هوشبهرهاى استدلال سيال، دانش، استدلال كمّى، يردازش

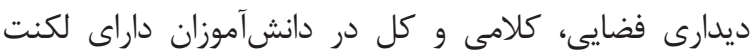

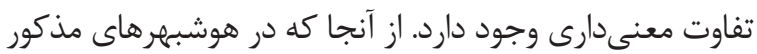

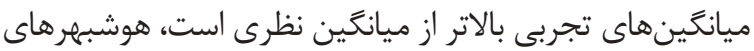

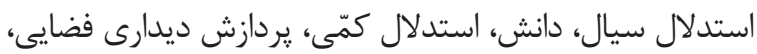

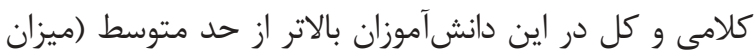

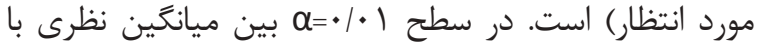

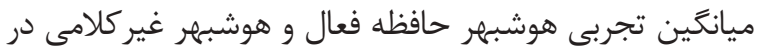

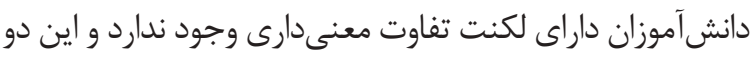
هوشبهر در اين دانشآموزان در حد متوسط جامعان است.

هدف از انجام اين يروهش بررسى كنشهاى شناختى

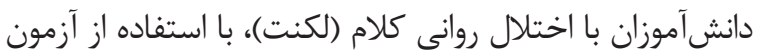

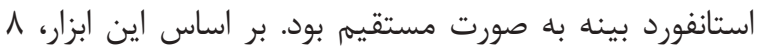

فضايى كلامى در دانشآموزان داراى لكنت وجود دارد. بنابراين،

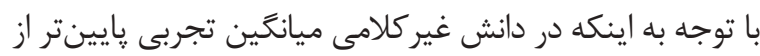

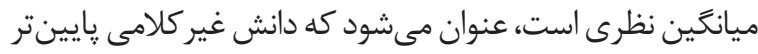

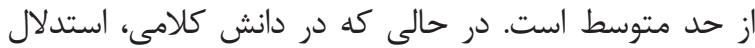

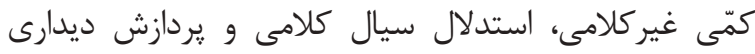

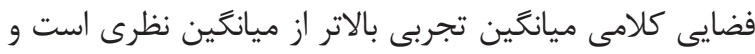

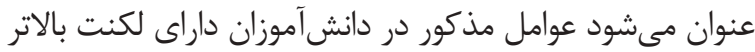

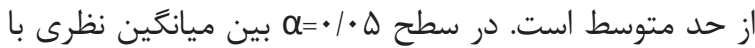

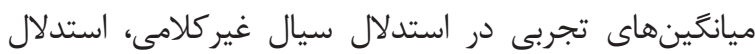

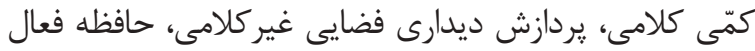

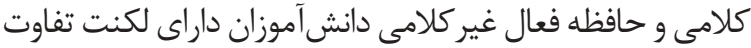

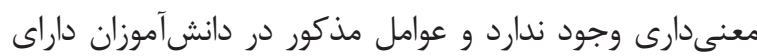
لكنت در حد متوسط (ميزان مورد انتظار) است.

بر اساس مقادير t بهدستآمده مىتوان كفت كه در سطح

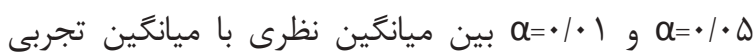



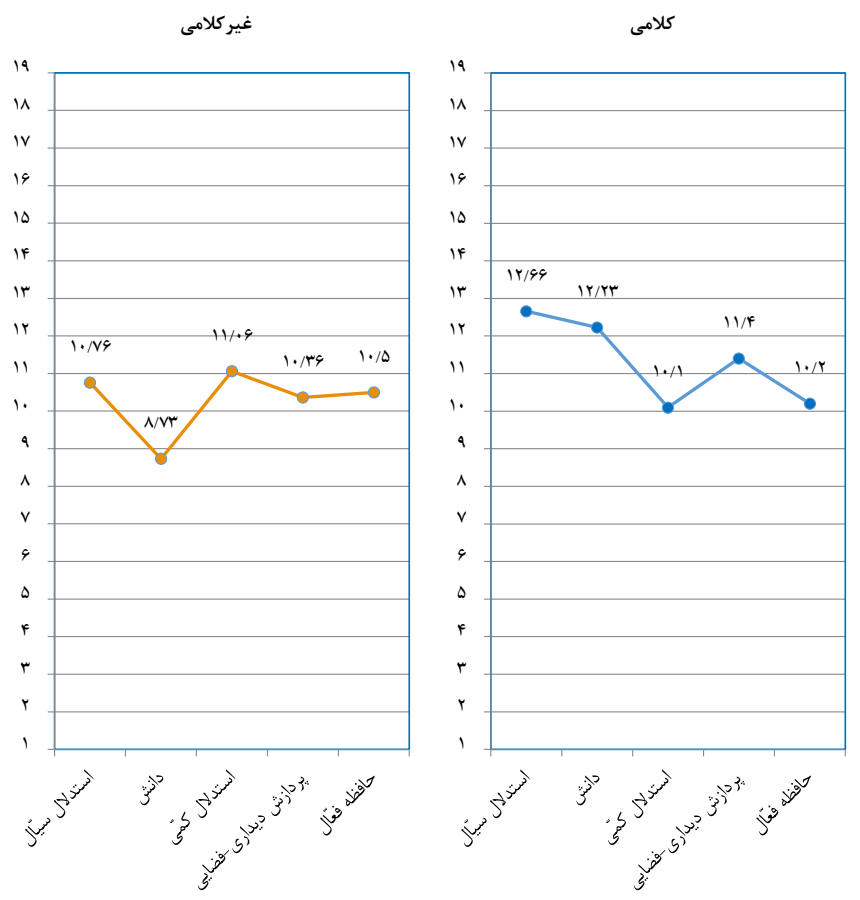

توانبخننى

تصوير ا. نيمرخ نمرات تراز خردهآزمونها در داتُش آموزان داراى لكنت

و هوشبهر كل محاسبه شده است.

براساس مقادير موجود در جدول شماره ا و تصاوير شماره

هوشبهر ازجمله له هوشبهر عوامل شناختى سازنده هوش شامل

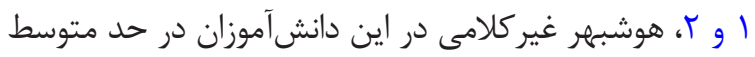

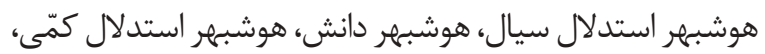

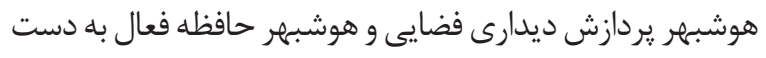

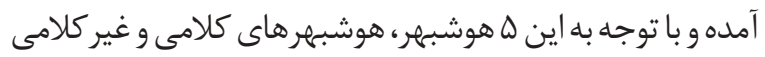

هوشبهر هاى هشتكانه

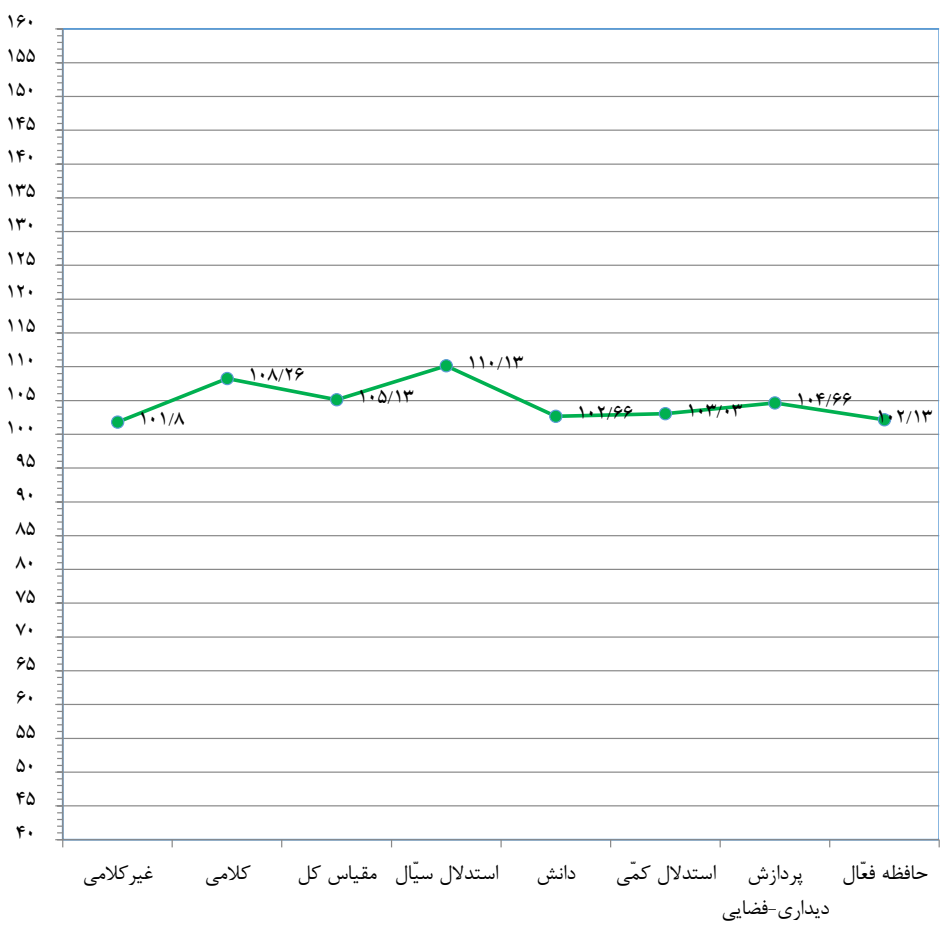

توانبخننى تصوير r. نيمرخ نمرات هوشبهر دانش آموزان داراى لكنث 
از آنجا كه تاكنون تحقيقاتى ييرامون رابطه لكنت و هوشبهرهاى

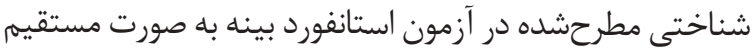

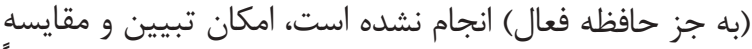

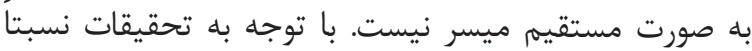

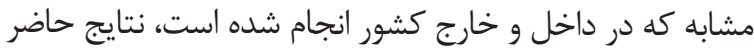

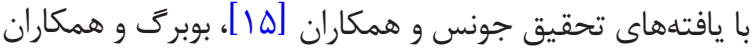

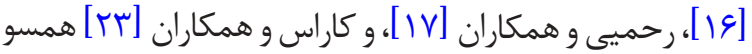

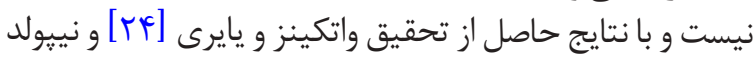

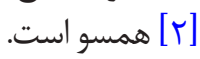

اين يزوهش با محدوديتهايى مواجه بود. از آنجا كه متغير

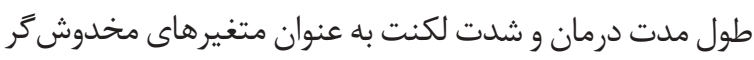

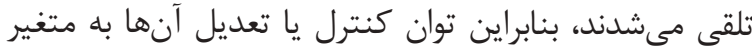

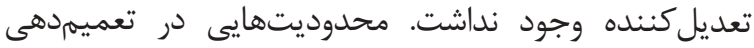

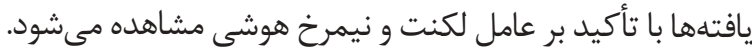

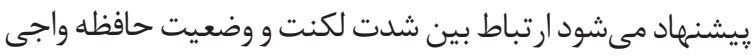

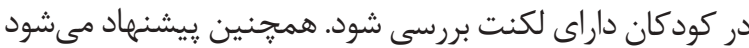

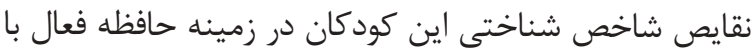
نقايص بزر كسالان داراى لكنت مقايسه شود.

\section{نتيجليرى}

بر اساس يافتههاى يزوهش نتيجه مى كيريم كه هوشبهر

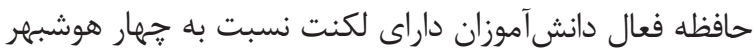

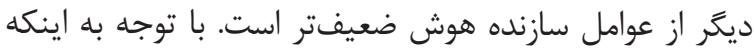

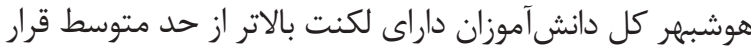

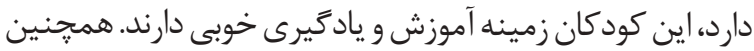

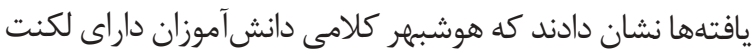

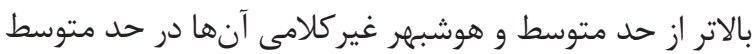

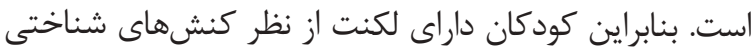

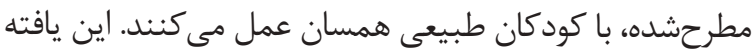

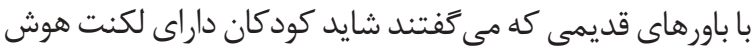

كمترى داشته باشند، مغايرت دارد و مخالف آن ديد داركاه است.

$$
\text { تشكر و قدر بأي }
$$

إين مقاله از پايان نامه كارشناسى ارشد نويسنده سوم

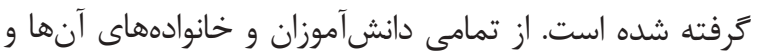

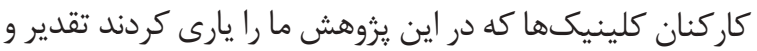

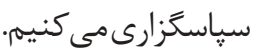

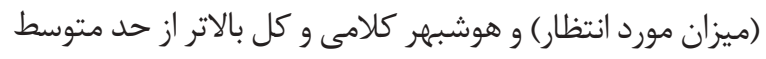

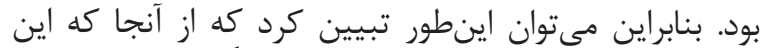

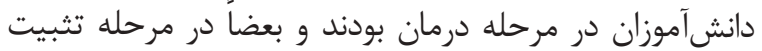

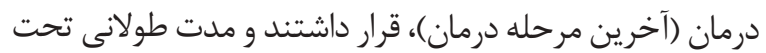

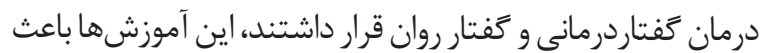

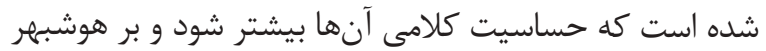

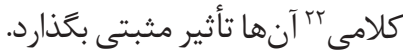

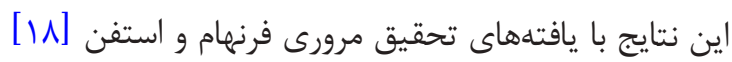

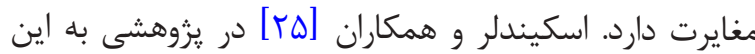

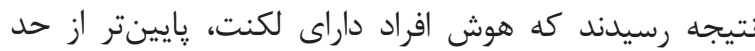

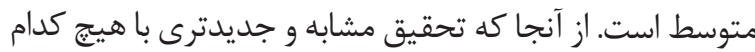

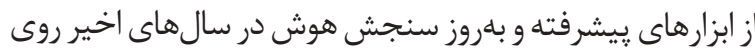

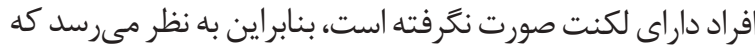

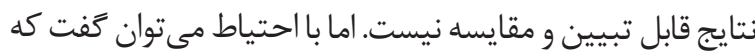

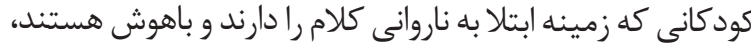
بيشتر در معرض لكنت قرار دارند.

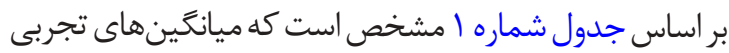

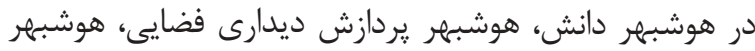

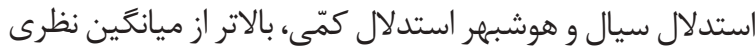

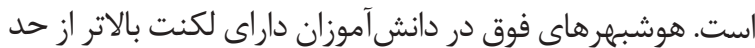

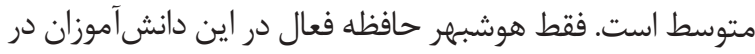

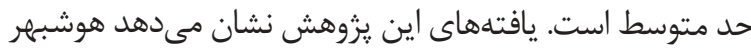

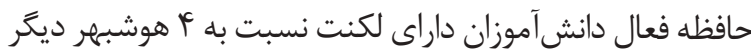

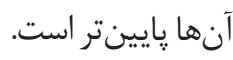

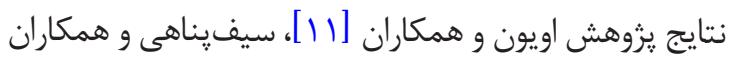

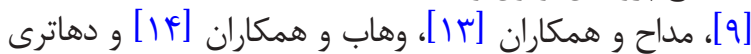

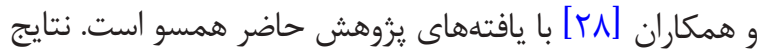

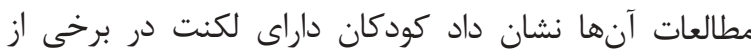

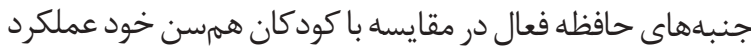

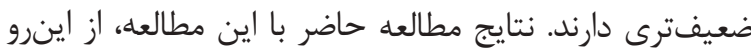

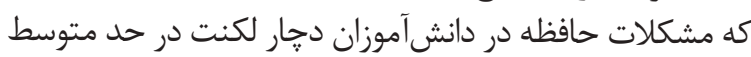

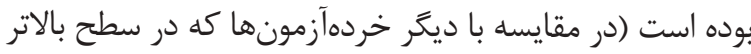

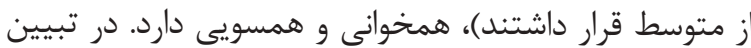

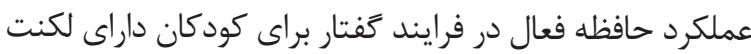

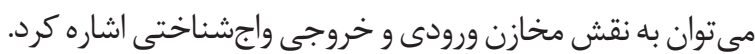

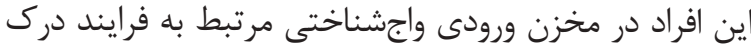

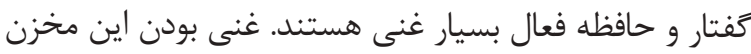

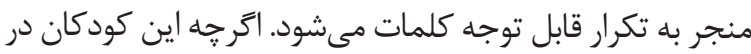

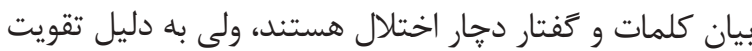

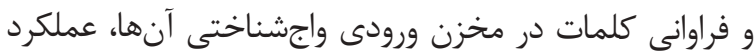

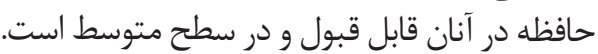




\section{References}

[1] Ward D. Stuttering and cluttering: Frameworks for understanding and treatment. Abingdon: Routledge. 2006.

[2] Nippold MA. Stuttering and language ability in children: Questioning the connection. American Journal of Speech-Language Pathology. 2012; 21(3):183-96. [DOI:10.1044/1058-0360(2012/11-0078)]

[3] Healey EC. The CALMS: A multidimensional approach to assessing and treatment school-age children who stutter. The Bulletin. 2013; 11(1):1-22.

[4] McDowell S, Whyte J, D’Esposito M. Working memory impairments in traumatic brain injury: Evidence from a dual-task paradigm. Neuropsychologia. 1997; 35(10):1341-53. [DOI:10.1016/S00283932(97)00082-1]

[5] Afrooz Gh, Kamkary K. [Psychometric principles and intelligence: Tehran University Stanford-Binan Intelligence Test (Persian)]. Tehran: University of Tehran Press; 2009.

[6] Aminloo S, Kamkary K, Shokrzadeh S. [The concurrent validity of the new version of the Tehran-Stanford-Binet Intelligence Scale with the Wechsler Intelligence Scale for children-revised (Persian)]. Journal of Exceptional Education. 2012; 13(7):50-61.

[7] Baddely A. Working memory and language: an overview. Journal of Communication Disorders. 2003; 36(3):189-208. [DOI:10.1016/ S0021-9924(03)00019-4]

[8] Basi M, Farazi M, Bakhshi E. Evaluation of effects of Gradual Increase Length and Complexity of Utterance (GILCU) treatment method on the reduction of dysfluency in school-aged children with stuttering. Iranian Rehabilitation Journal. 2016; 14(1):59-62 [DOI:10.15412/J.IRJ.08140109]

[9] Saifpanahi S, Sobhani Rad D, Afzali M, Izanloo S, Mardani N, Gholamian M. [An investigation of the correlation between phonological and visual working memory with severity of stuttering in 6-12 yearsold children (Persian)]. Journal of Paramedical Sciences \& Rehabilitation. 2015; 4(4):20-6.

[10] Baezzat F, Moradi M, Motaghedifard M. The effect of phonological awareness on the auditory memory in students with spelling problems. Iranian Rehabilitation Journal. 2018; 16(1):83-9. [DOI:10.29252/nrip.iri.16.1.83]

[11] Oyoun HA, El Dessouky H, Shohdi S, Fawzy A. Assessment of working memory in normal children and children who stutter. Journal of American Science. 2010; 6(11):562-6.

[12] Vameghi R, Sajedi F, Yadegari F, Zarifian T, Shahshahanipour S, Hatamizadeh N, et al. Production of a protocol on early intervention for speech and language delays in early childhood: A novice experience in Iran (Persian)]. Archives of Rehabilitation. 2016; 16(4):37481.

[13] Maddah M, AzadFallah P, Salmani M, RasoolZade Tabatabayi K. [Phonological working memory in children with normal non-fluency (Persian)]. Journal of Clinical Psychology. 2011; 3(3):1-6.

[14] Vahab M, Shojaei K, Ahmadi A, Nasiri M. [Phonological working memory in 4-8 year-old Persian children who stutter (Persian)]. Journal of Rehabilitation Sciences and Research. 2015; 1(4):92-6.

[15] Jones RD, White AJ, Lawson KH, Anderson T]. Visuoperceptual and visuomotor deficits in developmental stutterers: An exploratory study. Human Movement Science. 2002; 21(5):603-9. [DOI:10.1016/ S0167-9457(02)00165-3]

[16] Boberg E, Yeudall LT, Schopflocher D, Bo-Lassen P. The effect of an intensive behavioral program on the distribution of EEG alpha power in stutterers during the processing of verbal and visuospatial information. Journal of Fluency Disorders. 1983; 8(3):245-63. [DOI:10.1016/0094-730X(83)90038-4]

[17] Rahimi SS Farazi M, Darouie A, Bakhshi E, Abdi S, Valinejad V, Tiemori M. [Comparison the quality of life among adults with and without stuttering: An emohasis on the severity of stuttering (Persian)]. Journal of Rehabilitation. 2016; 17(4):300-6. [DOI:10.21859/ jrehab-1704300]

[18] Furnham A, Davis S. Involvement of social factors in stuttering: A review and assessment of current methodology. Stammering research: An Online Journal Published by the British Stammering Association. 2004; 1(2):112-22. [PMID] [PMCID]

[19] Farazi M, Sajedi F. [Speech and language intervention in children with speech disorder (Persian)]. Tehran: University of Social Welfare and Rehabilitation Sciences; 2016.

[20] Farazi M, Sajedi F. [Stuttering in children (Persian)]. Speech and Language Pathology. 2014; 1(4):62-9.

[21] Farazi M, Gholami Tehrani L, Khodabakhshi Kolaee A, Shemshadi H, Rahgozar M. The effect of a combination of cognitive therapy and speech in stutterers (Persian)]. Thought \& Behavior in Clinical Psychology. 2014; 8(30):37-46.

[22] St Clare T, Menzies RG, Onslow M, Packman A, Thompson, R, Block, S. Unhelpful thoughts and beliefs linked to social anxiety in stuttering: Development of a measure. International Journal of Language \& Communication Disorders. 2009; 44(3):13. [DOI:10.1080/13682820802067529] [PMID]

[23] Karrass J, Walden TA, Conture EG, Graham CG, Arnold HS, Hartfield KN, Schwenk KA. Relation of emotional reactivity and regulation to childhood stuttering. Journal of Communication Disorders. 2006; 39(6):402-26. [DOI:10.1016/j.jcomdis.2005.12.004] [PMID] [PMCID]

[24] Watkins RV, Yairi E. Language production abilities of children who stuttering persisted or recovered. Journal of Speech, Language, and Hearing Research. 1997; 40(2):385-99. [DOI:10.1044/jslhr.4002.385]

[25] Schwenk KA, Conture EG, Walden TA. Reaction to background stimulation of preschool children who do and do not stutter. Journal of Communication Disorders. 2007; 40(2):129-41. [DOI:10.1016/j. jcomdis.2006.06.003] [PMID] [PMCID]

[26] Yadegari F, Salehi A. [Diffevential diagnosis of frimary stuttering and normal nonfluency in children referring to Saba Clinic (Persian)]. Archives of Rehabilitation. 2003; 4(3):45-8.

[27] Eggers K, De Nil, Vandenberg BR. Inhibitory control in childhood stuttering. Journal of Fluency Disorders. 2007; 38(1):1-13. [DOI:10.1016/j.jfludis.2012.10.001] [PMID]

[28] Dhatri SD, Ajith U, Santosh M. Camparison of working memory abilities in adults who do and not stutter. Journal of Indian Speech, Language \& Hearing Association. 2017; 31(2):42-7. [DOI:10.4103/ jisha.JISHA_5_17] 